\title{
Chapter 6 \\ Lattice methods and the nuclear few- and many-body problem
}

Dean Lee

\begin{abstract}
We begin with a brief overview of lattice calculations using chiral effective field theory and some recent applications. We then describe several methods for computing scattering on the lattice. After that we focus on the main goal, explaining the theory and algorithms relevant to lattice simulations of nuclear few- and many-body systems. We discuss the exact equivalence of four different lattice formalisms, the Grassmann path integral, transfer matrix operator, Grassmann path integral with auxiliary fields, and transfer matrix operator with auxiliary fields. Along with our analysis we include several coding examples and a number of exercises for the calculations of few- and many-body systems at leading order in chiral effective field theory.
\end{abstract}

\subsection{Introduction}

This chapter builds upon the general overview of lattice methods for effective field theory of the previous chapter. We discuss the theory and algorithms used in lattice simulations of nuclear few and many body systems. We show the exact equivalence of the Grassmann path integral, transfer matrix operator, Grassmann path integral with auxiliary fields, and transfer matrix operator with auxiliary fields. Along with our analysis we include several coding examples and a number of exercises for the calculations of few- and many-body systems at leading order in chiral effective field theory.

Effective field theory (EFT) provides a theoretical framework for organizing low-energy interactions in powers of particle momenta. Chiral effective field theory applies this framework to the low-energy interactions of protons and neutrons while explicitly including the interactions of pions [1-9]. Pions are qualitatively different from other mesons since they become massless in the limit of massless quarks, thereby producing long-range exchange interactions. The low-energy expansion of chiral EFT is organized in powers of $Q$, where $Q$ denotes the typical momentum of the nucleons as well as explicit factors of the pion mass. The most important interactions are called leading order (LO) or $O\left(Q^{0}\right)$. The next most important contributions are next-to-leading order (NLO) or $O\left(Q^{2}\right)$. The terms after this are next-to-next-to-leading order (NNLO) or $O\left(Q^{3}\right)$, and so on.

Lattice EFT refers generally to lattice simulations based upon the framework of effective field theory. There are a few reviews in the literature which discuss current methods used in lattice effective field theory [10,11] as well as the discussion in the previous chapter of

Dean Lee

Department of Physics, North Carolina State University, Raleigh, NC 27695, USA, e-mail: dean_lee@ncsu.edu 
this volume. Many different phenomena can be studied in lattice EFT using the same lattice action. In principle all systematic errors are introduced up front when defining the lowenergy effective theory, as opposed to the particular computational scheme used to calculate observables.

Lattice EFT has been aided by efficient lattice methods developed for lattice QCD and condensed matter applications. The methods include Markov Chain Monte Carlo techniques, auxiliary fields [12]13], pseudofermion methods [14], and non-local updating schemes such as the hybrid Monte Carlo algorithm [15-17]. Lattice EFT was first used in studies of infinite nuclear matter [18] and infinite neutron matter with and without explicit pions [19] 22]. The method has also been used to study light nuclei in pionless EFT [23] and chiral EFT at leading order [24]. There have been further studies of neutron matter [25 27] and light nuclei [28 29], and there have been several applications to nuclear structure and nuclear clustering [30-35] as well as recent work on nuclear scattering and reactions [36-38].

\subsection{Recent Applications}

We review here several recent applications of lattice effective field theory to nuclear systems. In Ref. [34], the first $a b$ initio evidence is presented for a tetrahedral alpha-cluster structure of the ground state of ${ }^{16} \mathrm{O}$. The first excited $0^{+}$state of ${ }^{16} \mathrm{O}$ is found to be a planar or square arrangement of alpha clusters. The evidence for these geometric arrangements come from the strong overlap between nuclear states and initial state configurations with these alphacluster geometries.

In Table 6.1 we presented the energies of the low-lying even parity states of oxygen-16. The columns labeled "LO(2N)" and "NNLO(2N)" show the energies at each order using the twonucleon force only. The column labeled " $+3 \mathrm{~N}$ " also includes the $3 \mathrm{NF}$, which first appears at

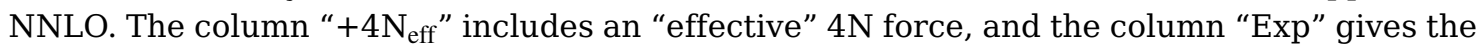
empirical energies. This "effective" $4 \mathrm{~N}$ force was introduced in Ref. [33] as a proxy measure of unknown systematic errors responsible for overbinding in lattice chiral effective field theory calculations with increasing numbers of nucleons. This tendency towards overbinding has also been noted in other nuclear structure calculations [39,40].

Table 6.1 Lattice results and experimental energies for the lowest even-parity states of ${ }^{16} \mathrm{O}$ in $\mathrm{MeV}$. The errors include statistical Monte Carlo errors and uncertainties due to the extrapolation to infinite Euclidean time.

\begin{tabular}{r|r|rrr|c}
$J_{n}^{p}$ & LO (2N) & NNLO (2N) & \multicolumn{1}{c}{$+3 \mathrm{~N}$} & \multicolumn{1}{c}{$+4 \mathrm{~N}_{\mathrm{eff}}$} & \multicolumn{1}{c}{$\operatorname{Exp}$} \\
\hline \hline $0_{1}^{+}$ & $-147.3(5)$ & $-121.4(5)$ & $-138.8(5)$ & $-131.3(5)$ & -127.62 \\
$0_{2}^{+}$ & $-145(2)$ & $-116(2)$ & $-136(2)$ & $-123(2)$ & -121.57 \\
$2_{1}^{+}$ & $-145(2)$ & $-116(2)$ & $-136(2)$ & $-123(2)$ & -120.70
\end{tabular}

In order to understand the source of this overbinding, the problem was revisited again in Ref. [35]. In that work numerical evidence from ab initio lattice simulations showed that the problem appears related to the fact that the nuclear forces reside near a quantum phase transition. Using lattice effective field theory, Monte Carlo simulations were performed for systems with up to twenty nucleons. For even and equal numbers of protons and neutrons, a first-order transition was found at zero temperature from a Bose-condensed gas of alpha particles to a nuclear liquid. Whether one has an alpha-particle gas or nuclear liquid is determined by the strength of the alpha-alpha interactions, and the alpha-alpha interactions depend on the strength and locality of the nucleon-nucleon interactions. This insight is useful 
in improving calculations of nuclear structure and important astrophysical reactions involving alpha capture on nuclei. These findings also provide a tool to probe the structure of alpha cluster states such as the Hoyle state responsible for the production of carbon in red giant stars and point to a connection between nuclear states and the universal physics of bosons at large scattering length.

Processes such as the scattering of alpha particles, the triple-alpha reaction, and alpha capture play an important role in stellar nucleosynthesis. In particular, alpha capture on carbon determines the ratio of carbon to oxygen during helium burning and impacts the following carbon, neon, oxygen, and silicon burning stages. In these reactions the elastic scattering of alpha particles and alpha-like nuclei (nuclei with even and equal numbers of protons and neutrons) are important for understanding background and resonant scattering contributions. In Ref. [38] the first $a b$ initio calculations of the scattering of two alpha particles were performed using a technique called the adiabatic projection method. These calculations represent a significant algorithmic improvement since the calculations presented in Ref. [38] scale roughly quadratically with the number of nucleons and opens a gateway to scattering and reactions involving heavier nuclei.

\subsection{Scattering on the lattice}

At any given order in the chiral EFT expansion, there will be short-range interaction coefficients which depend on the chosen regularization of the large-momentum divergences. On the lattice this regularization is provided by the lattice spacing, unless some additional regularization is applied to the lattice interactions. In order to set the values of the short-range two-nucleon interaction coefficients, we make a comparison of nucleon-nucleon scattering on the lattice with experimental scattering data. The extension to three-nucleon interaction coefficients is also required at NNLO, and that procedure on the lattice has been discussed in Ref. [28]

As discussed in the previous chapter, Lüscher [41-43] has shown that the finite-volume energy levels for a two-body system in a periodic cubic box are related to the infinite-volume scattering matrix. While the method is very useful at low momenta, it can become less accurate at higher momenta and higher orbital angular momenta. Also spin-orbit coupling and partial-wave mixing are difficult to measure accurately using Lüscher's method due to scattering artifacts produced by the cubic periodic boundary. An alternative approach has been developed to measure phase shifts for particles on the lattice using a spherical wall boundary [44. 45].

In this approach, a hard spherical wall boundary is imposed on the relative separation between the two particles. This wall is placed at some chosen radius $R_{\text {wall }}$, and it removes copies of the interactions produced by the periodic lattice. Working in the center-of-mass frame, we solve the time-independent Schrödinger equation as a function of the relative separation between the particles and compute spherical standing waves which vanish at $r=R_{\text {wall }}$. At values of $r$ beyond the range of the interaction, the spherical standing waves can be written as a superposition of products of spherical harmonics and spherical Bessel functions,

$$
\left[\cos \delta_{\ell} \cdot j_{\ell}(k r)-\sin \delta_{\ell} \cdot y_{\ell}(k r)\right] Y_{\ell, \ell_{z}}(\theta, \phi) .
$$

Here $k$ is the relative momentum between the scattering particles, and $\delta_{\ell}$ is the phase shift for partial wave $\ell$. We can extract $k$ from the energy of the standing wave, and the phase shift $\delta_{\ell}$ is determined by setting the wave function in Eq. (6.1) to zero at the wall boundary. 
When the total intrinsic spin of the two nucleons is nonzero, spin-orbit coupling generates mixing between partial waves. In this case the standing wave at the wall boundary is decomposed into spherical harmonics and coupled-channel equations are solved to extract the phase shifts and mixing angles. The spherical wall method was used to calculate phase shifts and mixing angle for low-energy nucleon-nucleon scattering [25]. Recently the spherical wall approach has been improved in accuracy and computational efficiency [46]. In the improved approach one projects onto spherical harmonics $Y_{\ell, \ell_{z}}$ with angular momentum quantum numbers $\ell, \ell_{z}$. In this manner one constructs radial position states for a given partial wave,

$$
|r\rangle^{\ell, \ell_{z}}=\sum_{\mathbf{r}^{\prime}} Y_{\ell, \ell_{z}}\left(\hat{\mathbf{r}}^{\prime}\right) \delta_{r,\left|\mathbf{r}^{\prime}\right|}\left|\mathbf{r}^{\prime}\right\rangle
$$

We require that $r$ is less than half the box length $L / 2$. Using this technique we are essentially constructing a radial position basis for each partial wave.

It is also useful to introduce auxiliary potentials in the region lying just in front of the spherical wall boundary [46]. The auxiliary potential is a spherical attractive well that is positioned in front of the spherical wall boundary. We can tune to any scattering energy by adjusting the depth of the well. For systems with partial wave mixing due to spin-orbit coupling, we also include a Hermitian but imaginary off-diagonal auxiliary potential for the two coupled channels. This breaks time reversal symmetry, and the resulting standing wave solutions now have both real and imaginary parts that are linearly independent. From the real and imaginary solutions one can determine the scattering phase shifts and mixing angle at any given value of the scattering energy.

This spherical wall approach has been used together with a technique called the adiabatic projection method to study nuclear scattering and reactions on the lattice. The adiabiatic projection method [35, 38, 47, 49] is a general framework that produces a low-energy effective theory for clusters of particles which becomes exact in the limit of large projection time. For the case of two-cluster scattering, we consider a set of two cluster states $|\mathbf{R}\rangle$ labeled by the spatial separation vector $\mathbf{R}$. The initial wave functions are wave packets which, for large $|\mathbf{R}|$, factorize into a product of two individual clusters,

$$
|\mathbf{R}\rangle=\sum_{\mathbf{r}}|\mathbf{r}+\mathbf{R}\rangle_{1} \otimes|\mathbf{r}\rangle_{2}
$$

The summation over $\mathbf{r}$ is required to produce states with total momentum equal to zero. We bin the initial cluster states together according to radial distance and angular momentum. In this manner, we form radial position states with projected angular momentum quantum numbers, which we label $|R\rangle^{\ell, \ell_{z}}$.

The next step is to multiply by powers of the transfer matrix in order to form "dressed" cluster states. This produces states that approximately span the set of low-energy clustercluster scattering states in our periodic box. We discuss the transfer matrix formalism in detail later in this chapter. After $n_{t}$ time steps, we have the dressed cluster states

$$
|R\rangle_{n_{t}}^{\ell, \ell_{z}}=M^{n_{t}}|R\rangle^{\ell, \ell_{z}} .
$$

These dressed cluster states are then used to compute matrix elements of the transfer matrix $M$,

$$
\left[M_{n_{t}}\right]_{R^{\prime}, R}^{\ell, \ell_{z}}={ }_{n_{t}}^{\ell, \ell_{z}}\left\langle R^{\prime}|M| R\right\rangle_{n_{t}}^{\ell, \ell_{z}} .
$$

Since such states are not orthogonal, we also compute a norm matrix

$$
\left[N_{n_{t}}\right]_{R^{\prime}, R}^{\ell, \ell_{z}}=\stackrel{\ell, \ell_{2}}{n_{t}}\left\langle R^{\prime} \mid R\right\rangle_{n_{t}}^{\ell, \ell_{z}} .
$$

The "radial adiabatic transfer matrix" is defined as the matrix product 


$$
\left[M_{n_{t}}^{a}\right]_{R^{\prime}, R}^{\ell, \ell_{z}}=\left[N_{n_{t}}^{-\frac{1}{2}} M_{n_{t}} N_{n_{t}}^{-\frac{1}{2}}\right]_{R^{\prime}, R}^{\ell, \ell_{z}}
$$

and the scattering phase shifts can then be determined from the standing waves of the radial adiabatic transfer matrix.

\subsection{Lattice formalisms}

Throughout our discussion of the lattice formalism we use dimensionless parameters and operators corresponding with physical values times the appropriate power of the spatial lattice spacing $a$. In our notation the three-component integer vector $\mathbf{n}$ labels the lattice sites of a three-dimensional periodic lattice with dimensions $L^{3}$. The spatial lattice unit vectors are denoted $\hat{\mathbf{I}}=\hat{\mathbf{1}}, \hat{\mathbf{2}}, \hat{\mathbf{3}}$. We use $n_{t}$ to label lattice steps in the temporal direction, and $L_{t}$ denotes the total number of lattice time steps. The temporal lattice spacing is given by $a_{t}$, and $\alpha_{t}=a_{t} / a$ is the ratio of the temporal to spatial lattice spacing. We also define $h=\alpha_{t} /(2 m)$, where $m$ is the nucleon mass in lattice units. In Fig. 6.1 we show a diagram of the four different but exactly equivalent lattice formulations that we discuss, the Grassmann path integral, transfer matrix operator, Grassmann path integral with auxiliary fields, and transfer matrix operator with auxiliary fields.

\subsubsection{Grassmann path integral}

We define the lattice action starting from the lattice Grassmann path integral action without auxiliary fields. This is the simplest formulation in which to derive the lattice Feynman rules. We let $c$ and $c^{*}$ be anticommuting Grassmann fields for the nucleons. In our notation $c$ is a column vector composed of the spin-isospin nucleon degrees of freedom $c_{i}$, while $c^{*}$ is a row vector of the components $c_{i}^{*}$. The Grassmann fields are periodic with respect to the spatial extent of the $L^{3}$ lattice,

$$
\begin{aligned}
& c_{i}\left(\mathbf{n}+L \hat{1}, n_{t}\right)=c_{i}\left(\mathbf{n}+L \hat{2}, n_{t}\right)=c_{i}\left(\mathbf{n}+L \hat{3}, n_{t}\right)=c_{i}\left(\mathbf{n}, n_{t}\right), \\
& c_{i}^{*}\left(\mathbf{n}+L \hat{1}, n_{t}\right)=c_{i}^{*}\left(\mathbf{n}+L \hat{2}, n_{t}\right)=c_{i}^{*}\left(\mathbf{n}+L \hat{3}, n_{t}\right)=c_{i}^{*}\left(\mathbf{n}, n_{t}\right),
\end{aligned}
$$

and antiperiodic along the temporal direction,

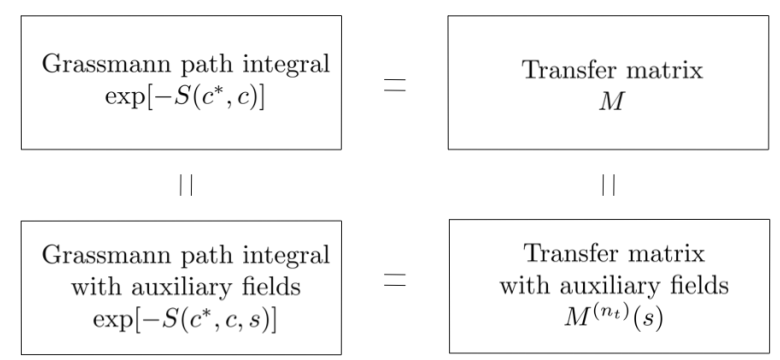

Fig. 6.1 A schematic diagram of the different lattice formulations, namely, the Grassmann path integral, transfer matrix operator, Grassmann path integral with auxiliary fields, and transfer matrix operator with auxiliary fields. 


$$
\begin{gathered}
c_{i}\left(\mathbf{n}, n_{t}+L_{t}\right)=-c_{i}\left(\mathbf{n}, n_{t}\right), \\
c_{i}^{*}\left(\mathbf{n}, n_{t}+L_{t}\right)=-c_{i}^{*}\left(\mathbf{n}, n_{t}\right) .
\end{gathered}
$$

We write $D c D c^{*}$ as shorthand for the integral measure,

$$
D c D c^{*}=\prod_{\mathbf{n}, n_{t}, i} d c_{i}\left(\mathbf{n}, n_{t}\right) d c_{i}^{*}\left(\mathbf{n}, n_{t}\right) .
$$

We use the usual convention for Grassmann integration,

$$
\begin{gathered}
\int d c_{i}\left(\mathbf{n}, n_{t}\right)=\int d c_{i}^{*}\left(\mathbf{n}, n_{t}\right)=0 \\
\int d c_{i}\left(\mathbf{n}, n_{t}\right) c_{i}\left(\mathbf{n}, n_{t}\right)=\int d c_{i}^{*}\left(\mathbf{n}, n_{t}\right) c_{i}^{*}\left(\mathbf{n}, n_{t}\right)=1 \quad(\text { no sum on } i) .
\end{gathered}
$$

We consider the Grassmann path integral

$$
\mathscr{Z}=\int D c D c^{*} \exp \left[-S\left(c^{*}, c\right)\right]
$$

where the lattice action can be broken into a free part and interacting part,

$$
S\left(c^{*}, c\right)=S_{\text {free }}\left(c^{*}, c\right)+S_{\text {int }}\left(c^{*}, c\right) .
$$

The free part is the free non-relativistic nucleon action, which is

$$
S_{\text {free }}\left(c^{*}, c\right)=\sum_{\mathbf{n}, n_{t}} c^{*}\left(\mathbf{n}, n_{t}\right)\left[c\left(\mathbf{n}, n_{t}+1\right)-c\left(\mathbf{n}, n_{t}\right)\right]+\alpha_{t} \sum_{n_{t}} K^{\left(n_{t}\right)}\left(c^{*}, c\right),
$$

where

$$
K^{\left(n_{t}\right)}\left(c^{*}, c\right)=\sum_{k=0,1,2, \cdots}(-1)^{k} \frac{w_{k}}{2 m} \sum_{\mathbf{n}, \hat{\mathbf{I}}} c^{*}\left(\mathbf{n}, n_{t}\right)\left[c\left(\mathbf{n}+k \hat{\mathbf{l}}, n_{t}\right)+c\left(\mathbf{n}-k \hat{\mathbf{l}}, n_{t}\right)\right]
$$

and the hopping coefficients $w_{k}$ correspond to a hopping parameter expansion of the squared momentum,

$$
P^{2}(\mathbf{p})=2 \sum_{k=0,1,2, \cdots} \sum_{l=1,2,3}(-1)^{k} w_{k} \cos \left(k p_{l}\right) .
$$

The hopping coefficients are chosen to match the continuum relation

$$
P^{2}(\mathbf{p})=\mathbf{p}^{2}
$$

up to some chosen level of lattice discretization error. The hopping coefficients $w_{k}$ for a few different lattice actions are shown in Table 6.2.

Table 6.2 Hopping coefficients $w_{k}$ for several lattice actions

\begin{tabular}{llll}
\hline coefficient & standard & $O\left(a^{2}\right)$-improved & $O\left(a^{4}\right)$-improved \\
\hline$w_{0}$ & 1 & $5 / 4$ & $49 / 36$ \\
$w_{1}$ & 1 & $4 / 3$ & $3 / 2$ \\
$w_{2}$ & 0 & $1 / 12$ & $3 / 20$ \\
$w_{3}$ & 0 & 0 & $1 / 90$ \\
\hline
\end{tabular}




\subsubsection{Transfer matrix operator}

Let $a_{i}(\mathbf{n})$ and $a_{i}^{\dagger}(\mathbf{n})$ denote fermion annihilation and creation operators for the nucleon component $i$ at lattice site $\mathbf{n}$. The shorthand $a(\mathbf{n})$ represents a column vector of nucleon components $a_{i}(\mathbf{n})$, and $a^{\dagger}(\mathbf{n})$ represents a row vector of components $a_{i}^{\dagger}(\mathbf{n})$. We can write any Grassmann path integral with instantaneous interactions as the trace of a product of operators using the identity [50,51]

$$
\begin{aligned}
\operatorname{Tr}\left\{: F_{L_{t}-1}\left[a^{\dagger}\left(\mathbf{n}^{\prime}\right), a(\mathbf{n})\right]: \times \cdots \times: F_{0}\left[a^{\dagger}\left(\mathbf{n}^{\prime}\right), a(\mathbf{n})\right]:\right\} \\
=\int D c D c^{*} \exp \left\{\sum_{n_{t}=0}^{L_{t}-1} \sum_{\mathbf{n}, i} c_{i}^{*}\left(\mathbf{n}, n_{t}\right)\left[c_{i}\left(\mathbf{n}, n_{t}\right)-c_{i}\left(\mathbf{n}, n_{t}+1\right)\right]\right\} \\
\times \prod_{n_{t}=0}^{L_{t}-1} F_{n_{t}}\left[c^{*}\left(\mathbf{n}^{\prime}, n_{t}\right), c\left(\mathbf{n}, n_{t}\right)\right],
\end{aligned}
$$

where $c_{i}\left(\mathbf{n}, L_{t}\right)=-c_{i}(\mathbf{n}, 0)$.

Let us define the free non-relativistic lattice Hamiltonian

$$
H_{\text {free }}\left(a^{\dagger}, a\right)=\sum_{k=0,1,2, \cdots}(-1)^{k} \frac{w_{k}}{2 m} \sum_{\mathbf{n}, \hat{\imath}} a^{\dagger}(\mathbf{n})[a(\mathbf{n}+k \hat{\mathbf{l}})+a(\mathbf{n}-k \hat{\mathbf{l}})] .
$$

We write the interaction term as $H_{\text {int }}\left(a^{\dagger}, a\right)$, so that our total Hamiltonian is

$$
H\left(a^{\dagger}, a\right)=H_{\text {free }}\left(a^{\dagger}, a\right)+H_{\text {int }}\left(a^{\dagger}, a\right) .
$$

Using the correspondence Eq. 6.21, we can rewrite the path integral $\mathscr{Z}$ defined in Eq. 6.15 as a transfer-matrix partition function,

$$
\mathscr{Z}=\operatorname{Tr}\left(M^{L_{t}}\right),
$$

where $M$ is the normal-ordered transfer matrix operator

$$
M=: \exp \left[-H\left(a^{\dagger}, a\right) \alpha_{t}\right]: .
$$

Roughly speaking, the transfer matrix operator is the exponential of the Hamiltonian operator over one Euclidean lattice time step. In order to satisfy the identity Eq. (6.21), the exact definition of the transfer matrix is the normal-ordered exponential as defined in Eq. 6.25).

In this transfer matrix formalism, one can do simulations of nucleons using Monte Carlo, and this would essentially be a lattice version of diffusion or Green's function Monte Carlo [52]. Visually one can view the nucleons as interacting with each other while diffusing in space with each time step, as indicated in Fig. 6.2. At leading order in chiral effective field theory, the interactions include two independent $S$-wave contact interactions and the exchange of pions. We discuss these interactions in detail in the following.

\subsubsection{Grassmann path integral with auxiliary field}

We assume that there exists an integral relation that allows us to write $\exp \left[-S_{\text {int }}\left(c^{*}, c\right)\right]$ as an integral over auxiliary fields. The purpose of the auxiliary field transformation is to decouple the interactions among the nucleons. Instead the interactions will be between the nucleons and the auxiliary fields. 
Fig. 6.2 A sketch showing nucleons which evolve with each time step. At leading order in chiral effective field theory, the interactions include two contact interactions and the exchange of pions.

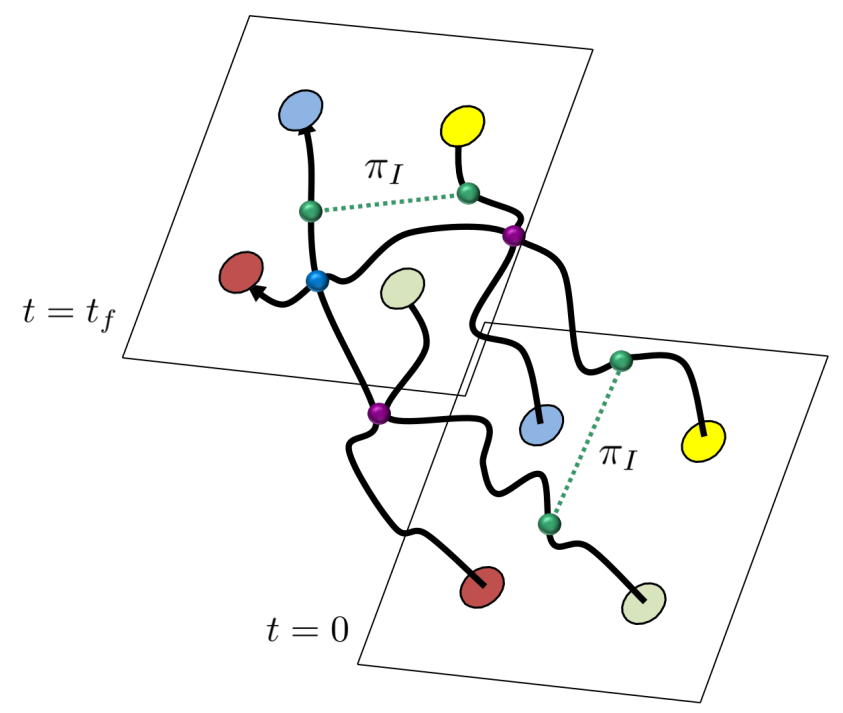

We illustrate using the interactions that appear at leading order in chiral effective field theory. For pedagogical purposes we discuss the simplest possible implementation of the leading order action on the lattice. We first consider a zero-range contact interaction which is independent of nucleon spin and isospin. The action has the form

$$
S_{\text {int }}^{C}\left(c^{*}, c\right)=\alpha_{t} \frac{C}{2} \sum_{\mathbf{n}, n_{t}}\left[c^{*}\left(\mathbf{n}, n_{t}\right) c\left(\mathbf{n}, n_{t}\right)\right]^{2} .
$$

We can write this as

$$
\exp \left[-S_{\text {int }}^{C}\left(c^{*}, c\right)\right]=\int D s \exp \left[-S_{s s}(s)-S_{S}\left(c^{*}, c, s\right)\right]
$$

for auxiliary field $s\left(\mathbf{n}, n_{t}\right)$, where

$$
\begin{gathered}
S_{s s}(s)=\frac{1}{2} \sum_{\mathbf{n}, n_{t}} s^{2}\left(\mathbf{n}, n_{t}\right) \\
S_{S}\left(c^{*}, c, s\right)=\sqrt{-C \alpha_{t}} \sum_{\mathbf{n}, n_{t}} s\left(\mathbf{n}, n_{t}\right) c^{*}\left(\mathbf{n}, n_{t}\right) c\left(\mathbf{n}, n_{t}\right) .
\end{gathered}
$$

In our definition of the integration measure $D s$, we include a factor of $1 / \sqrt{2 \pi}$ for each degree of freedom.

Next we consider an isospin-dependent contact interaction

$$
S_{\text {int }}^{C^{\prime}}\left(c^{*}, c\right)=\alpha_{t} \frac{C^{\prime}}{2} \sum_{\mathbf{n}, n_{t}, I}\left[c^{*}\left(\mathbf{n}, n_{t}\right) \tau_{I} c\left(\mathbf{n}, n_{t}\right)\right]^{2},
$$

where $\tau_{I}$ for $I=1,2,3$ are the Pauli matrices in isospin space. Then we can use

$$
\exp \left[-S_{\text {int }}^{C^{\prime}}\left(c^{*}, c\right)\right]=\int \prod_{I} D s_{I} \exp \left[-S_{S_{I} S_{I}}\left(s_{I}\right)-S_{S_{I}}\left(c^{*}, c, s_{I}\right)\right]
$$

for auxiliary fields $s_{I}\left(\mathbf{n}, n_{t}\right)$ where 
6 Lattice methods and the nuclear few- and many-body problem

$$
\begin{gathered}
S_{S_{I} S_{I}}\left(s_{I}\right)=\frac{1}{2} \sum_{\mathbf{n}, n_{t}, I} s_{I}^{2}\left(\mathbf{n}, n_{t}\right) \\
S_{S_{I}}\left(c^{*}, c, s_{I}\right)=\sqrt{-C^{\prime} \alpha_{t}} \sum_{\mathbf{n}, n_{t}, I} s_{I}\left(\mathbf{n}, n_{t}\right) c^{*}\left(\mathbf{n}, n_{t}\right) \tau_{I} c\left(\mathbf{n}, n_{t}\right) .
\end{gathered}
$$

Finally we work with the one-pion exchange potential (OPEP). In this case the pion acts much like the auxiliary fields. However there are also spatial correlations in the quadratic part of the pion action and a gradient coupling between the pions and nucleons. The one-pion exchange interaction on the lattice can written as

$$
\exp \left[-S_{\text {int }}^{\mathrm{OPEP}}\left(c^{*}, c\right)\right]=\int \prod_{I} D \pi_{I} \exp \left[-S_{\pi_{I} \pi_{I}}\left(\pi_{I}\right)-S_{\pi_{I}}\left(c^{*}, c, \pi_{I}\right)\right] .
$$

The free pion action is

$$
\begin{aligned}
S_{\pi_{I} \pi_{I}}\left(\pi_{I}\right)= & \frac{1}{2} \alpha_{t} m_{\pi}^{2} \sum_{\mathbf{n}, n_{t}, I} \pi_{I}^{2}\left(\mathbf{n}, n_{t}\right) \\
& +\frac{1}{2} \alpha_{t} \sum_{k=0,1,2, \cdots}(-1)^{k} w_{k} \sum_{\mathbf{n}, n_{t}, I, \hat{\mathbf{l}}} \pi_{I}\left(\mathbf{n}, n_{t}\right)\left[\pi_{I}\left(\mathbf{n}+k \hat{\mathbf{l}}, n_{t}\right)+\pi_{I}\left(\mathbf{n}-k \hat{\mathbf{l}}, n_{t}\right)\right],
\end{aligned}
$$

with the coefficient $w_{k}$ as defined in Table 6.2 and $m_{\pi}$ is the pion mass. At leading order we do not consider any isospin-breaking effects. The pion coupling to the nucleon is

$$
S_{\pi_{I}}\left(c^{*}, c, \pi_{I}\right)=\frac{g_{A} \alpha_{t}}{2 f_{\pi}} \sum_{\mathbf{n}, n_{t}, l, I} \Delta_{k} \pi_{I}\left(\mathbf{n}, n_{t}\right) c^{*}\left(\mathbf{n}, n_{t}\right) \sigma_{k} \tau_{I} c\left(\mathbf{n}, n_{t}\right),
$$

where $\sigma_{l}$ for $l=1,2,3$ are the Pauli matrices in spin space and

$$
\Delta_{l} \pi_{I}\left(\mathbf{n}, n_{t}\right)=\frac{1}{2} \sum_{k=1,2, \cdots}(-1)^{k-1} o_{k}\left[\pi_{I}\left(\mathbf{n}+k \hat{\mathbf{l}}, n_{t}\right)-\pi_{I}\left(\mathbf{n}-k \hat{\mathbf{l}}, n_{t}\right)\right],
$$

with coefficients $o_{k}$ corresponding to a hopping parameter expansion of the momentum,

$$
P\left(p_{l}\right)=\sum_{k=1,2, \cdots}(-1)^{k-1} o_{k} \sin \left(k p_{l}\right) \text {. }
$$

Here $g_{A}$ is the axial-vector coupling constant, and $f_{\pi}$ is the pion decay constant. The hopping coefficients can be chosen to match the continuum result

$$
P\left(p_{l}\right)=p_{l}
$$

The hopping coefficients $o_{k}$ for a few different lattice actions are shown in Table 6.3.

Table 6.3 Hopping coefficients $o_{k}$ for several lattice actions.

\begin{tabular}{llll}
\hline coefficient & standard & $O\left(a^{2}\right)$-improved & $O\left(a^{4}\right)$-improved \\
\hline$o_{1}$ & 1 & $4 / 3$ & $3 / 2$ \\
$o_{2}$ & 0 & $1 / 6$ & $3 / 10$ \\
$o_{3}$ & 0 & 0 & $1 / 30$ \\
\hline
\end{tabular}




\subsubsection{Transfer matrix operator with auxiliary field}

Using the equivalence in Eq. 6.21, we can write $\mathscr{Z}$ as the trace of a product of transfer matrix operators which depend on the auxiliary field,

$$
\mathscr{Z}=\int D s \prod_{I}\left(D s_{I} D \pi_{I}\right) \exp \left[-S_{s s}(s)-S_{S_{I} S_{I}}\left(s_{I}\right)-S_{\pi_{I} \pi_{I}}\left(\pi_{I}\right)\right] \operatorname{Tr}\left\{M^{\left(L_{t}-1\right)} \cdots M^{(0)}\right\} .
$$

The transfer matrix at time step $n_{t}$ is given by

$$
M^{\left(n_{t}\right)}=: \exp \left[-H^{\left(n_{t}\right)}\left(a^{\dagger}, a, s, s_{I}, \pi_{I}\right) \alpha_{t}\right]:
$$

where

$$
H^{\left(n_{t}\right)}\left(a^{\dagger}, a, s, s_{I}, \pi_{I}\right) \alpha_{t}=H_{\text {free }}\left(a^{\dagger}, a\right) \alpha_{t}+S_{s}^{\left(n_{t}\right)}\left(a^{\dagger}, a, s\right)+S_{s_{I}}^{\left(n_{t}\right)}\left(a^{\dagger}, a, s_{I}\right)+S_{\pi_{I}}^{\left(n_{t}\right)}\left(a^{\dagger}, a, \pi_{I}\right),
$$

and

$$
\begin{gathered}
S_{s}^{\left(n_{t}\right)}\left(a^{\dagger}, a, s\right)=\sqrt{-C \alpha_{t}} \sum_{\mathbf{n}} s\left(\mathbf{n}, n_{t}\right) a^{\dagger}(\mathbf{n}) a(\mathbf{n}), \\
S_{s_{I}}^{\left(n_{t}\right)}\left(a^{\dagger}, a, s_{I}\right)=\sqrt{-C^{\prime} \alpha_{t}} \sum_{\mathbf{n}, I} s_{I}\left(\mathbf{n}, n_{t}\right) a^{\dagger}(\mathbf{n}) \tau_{I} a(\mathbf{n}), \\
S_{\pi_{I}}^{\left(n_{t}\right)}\left(a^{\dagger}, a, \pi_{I}\right)=\frac{g_{A} \alpha_{t}}{2 f_{\pi}} \sum_{\mathbf{n}, k, I} \Delta_{k} \pi_{I}\left(\mathbf{n}, n_{t}\right) a^{\dagger}(\mathbf{n}) \sigma_{k} \tau_{I} a(\mathbf{n}) .
\end{gathered}
$$

\subsection{Projection Monte Carlo}

Let us consider a system with $A$ nucleons. We can create a general single-nucleon state using creation operators acting on the vacuum with coefficient function $f(\mathbf{n})$. We write $f(\mathbf{n})$ as a column vector in the space of nucleon spin and isospin components, and the single-nucleon state can be written as

$$
|f\rangle=\sum_{\mathbf{n}} a^{\dagger}(\mathbf{n}) f(\mathbf{n})|0\rangle
$$

For our projection Monte Carlo calculation we take our A-body initial state to be a Slater determinant of single nucleon states,

$$
\left|f_{1}, \cdots, f_{A}\right\rangle=\left[\sum_{\mathbf{n}} a^{\dagger}(\mathbf{n}) f_{1}(\mathbf{n})\right] \cdots\left[\sum_{\mathbf{n}} a^{\dagger}(\mathbf{n}) f_{A}(\mathbf{n})\right]|0\rangle .
$$

We use the same construction for the $A$-body final state.

For the purposes of coding the projection Monte Carlo calculation, it is convenient to view the identical nucleons as having a hidden index $j=1, \cdots, A$ that makes all of the nucleons distinguishable. If we antisymmetrize all physical states over this extra index then all physical observables are exactly recovered. So our initial state $\left|f_{1}, \cdots, f_{A}\right\rangle$ becomes

$$
\begin{aligned}
& \frac{1}{\sqrt{A !}} \sum_{P}\left[\sum_{\mathbf{n}} a_{[P(1)]}^{\dagger}(\mathbf{n}) f_{1}(\mathbf{n})\right] \ldots\left[\sum_{\mathbf{n}} a_{[P(A)]}^{\dagger}(\mathbf{n}) f_{A}(\mathbf{n})\right]|0\rangle \\
& \quad=\frac{1}{\sqrt{A !}} \sum_{P^{\prime}} \operatorname{sgn}\left(P^{\prime}\right)\left[\sum_{\mathbf{n}} a_{[1]}^{\dagger}(\mathbf{n}) f_{P^{\prime}(1)}(\mathbf{n})\right] \ldots\left[\sum_{\mathbf{n}} a_{[A]}^{\dagger}(\mathbf{n}) f_{P^{\prime}(A)}(\mathbf{n})\right]|0\rangle,
\end{aligned}
$$


Fig. 6.3 A sketch showing the worldline for a single nucleon with a background of pion fields and auxiliary fields.

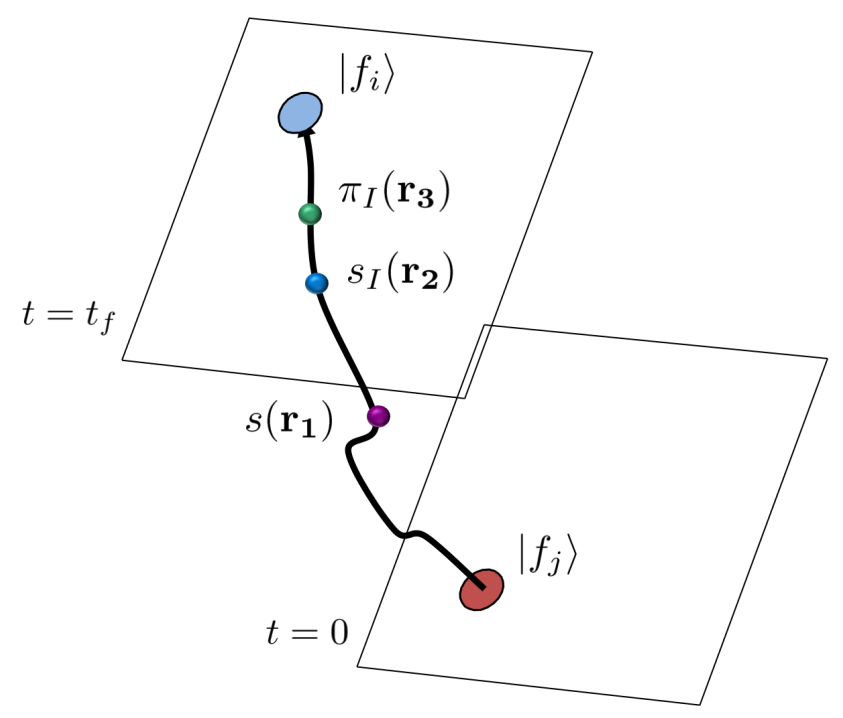

where the summations are over all permutations $P$, and sgn is the sign of the permutation. With these hidden indices our normal-ordered auxiliary-field transfer matrix $M^{\left(n_{t}\right)}$ becomes

$$
\left[1-H^{\left(n_{t}\right)}\left(a_{[1]}^{\dagger}, a_{[1]}, s, s_{I}, \pi_{I}\right) \alpha_{t}\right] \cdots\left[1-H^{\left(n_{t}\right)}\left(a_{[A]}^{\dagger}, a_{[A]}, s, s_{I}, \pi_{I}\right) \alpha_{t}\right]
$$

We see that the higher-order powers of the exponential vanish due to normal ordering.

In the projection Monte Carlo calculation we compute the amplitude

$$
Z\left(n_{t}\right)=\left\langle f_{1}, \cdots, f_{A}\left|M^{\left(n_{t}-1\right)} \cdots M^{(0)}\right| f_{1}, \cdots, f_{A}\right\rangle
$$

for $n_{t}=L_{t}$ and $n_{t}=L_{t}-1$. In the limit of large $L_{t}$ the amplitudes will be dominated by the state with the lowest energy $E_{0}$ and nonzero overlap with $\left|f_{1}, \cdots, f_{A}\right\rangle$. In this limit the ratio $Z\left(n_{t}\right) / Z\left(n_{t}-1\right)$ will converge to $\exp \left(-E_{0} \alpha_{t}\right)$ from above.

Each nucleon evolves as a particle in a fluctuating background of auxiliary fields and pion fields. The original interactions are reproduced after integrating over the fluctuating auxiliary and pion fields. For a simulation with $A$ nucleons, the amplitude for a given configuration of pion and auxiliary fields is proportional to the determinant of an $A \times A$ matrix M. The entries of $\mathbf{M}_{i j}$ are single nucleon worldline amplitudes for a nucleon starting at state $\left|f_{j}\right\rangle$ at $t=0$ and ending at state $\left|f_{i}\right\rangle$ at $t=t_{f}=L_{t} \alpha_{t}$. This is shown in Fig. 6.3.

In the following we show sample code 6.1 in the Fortran programming language which calculates the auxiliary-field transfer matrix multiplications on the left starting from the singlenucleon initial states. We show only the terms which arise from the free-nucleon transfer matrix and the auxiliary field $s$.

Listing 6.1 Sample code calculating the auxiliary-field transfer matrix multiplications on the left starting from the single-nucleon initial states

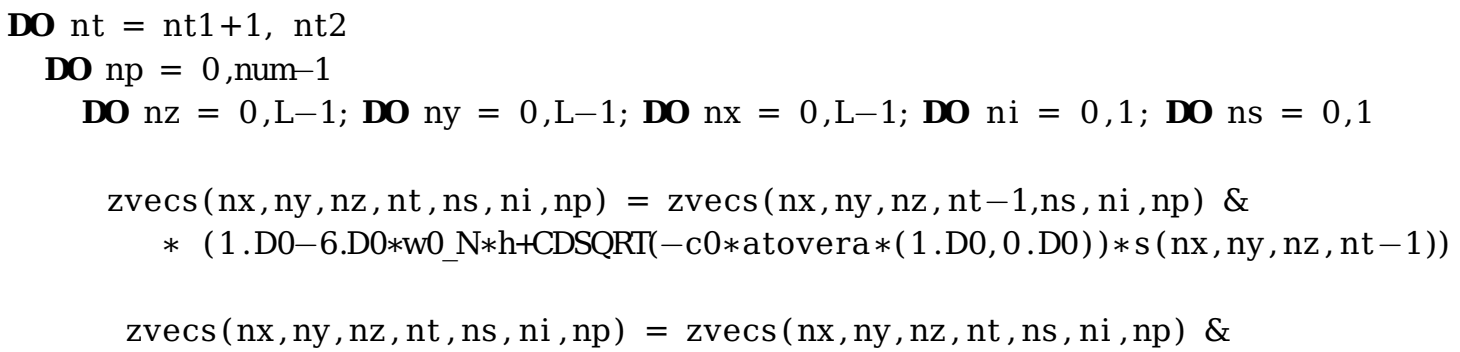


+ w1_N*h*zvecs (MOD(nx+1,L), ny, nz, nt-1,ns, ni,np) \&

+ w1_N*h*zvecs (MOD(nx-1+L, L), ny, nz, nt-1,ns, ni,np) \&

+ w1_N*h*zvecs ( $\mathrm{nx}, \mathrm{MOD}(\mathrm{ny}+1, \mathrm{~L}), \mathrm{nz}, \mathrm{nt}-1, \mathrm{~ns}, \mathrm{ni}, \mathrm{np}) \&$

+ w1_N*h*zvecs ( $\mathrm{nx}, \mathrm{MOD}(\mathrm{ny}-1+\mathrm{L}, \mathrm{L}), \mathrm{nz}, \mathrm{nt}-1, \mathrm{~ns}, \mathrm{ni}, \mathrm{np}) \&$

+ w1_N*h*zvecs ( $\mathrm{nx}, \mathrm{ny}, \mathrm{MOD}(\mathrm{nz}+1, \mathrm{~L}), \mathrm{nt}-1, \mathrm{~ns}, \mathrm{ni}, \mathrm{np}) \&$

$+\mathrm{w} 1 \_\mathrm{N} * \mathrm{~h} * \mathrm{zvecs}(\mathrm{nx}, \mathrm{ny}, \mathrm{MOD}(\mathrm{nz}-1+\mathrm{L}, \mathrm{L}), \mathrm{nt}-1, \mathrm{~ns}, \mathrm{ni}, \mathrm{np})$

IF (improveN $>=1$ ) THEN

zvecs $(\mathrm{nx}, \mathrm{ny}, \mathrm{nz}, \mathrm{nt}, \mathrm{ns}, \mathrm{ni}, \mathrm{np})=\operatorname{zvecs}(\mathrm{nx}, \mathrm{ny}, \mathrm{nz}, \mathrm{nt}, \mathrm{ns}, \mathrm{ni}, \mathrm{np}) \&$

- w2_N*h*zvecs (MOD(nx+2,L), ny, nz, nt-1,ns, ni , np) \&

- w2_N*h*zvecs (MOD(nx-2+L, L) , ny, nz, nt-1,ns, ni , np) \&

- w2_N*h*zvecs ( $\mathrm{nx}, \mathrm{MOD}(\mathrm{ny}+2, \mathrm{~L}), \mathrm{nz}, \mathrm{nt}-1, \mathrm{~ns}, \mathrm{ni}, \mathrm{np}) \&$

- w2_N*h*zvecs ( nx, MOD(ny-2+L, L) , nz, nt-1,ns , ni , np) \&

- w2_N*h*zvecs ( $\mathrm{nx}, \mathrm{ny}, \mathrm{MOD}(\mathrm{nz}+2, \mathrm{~L}), \mathrm{nt}-1, \mathrm{~ns}, \mathrm{ni}, \mathrm{np}) \&$

END IF

- w2_N*h*zvecs ( nx , ny ,MOD(nz-2+L, L) , nt-1,ns , ni ,np)

IF (improveN $==2$ ) $\mathbf{T H E N}$

zvecs $(\mathrm{nx}, \mathrm{ny}, \mathrm{nz}, \mathrm{nt}, \mathrm{ns}, \mathrm{ni}, \mathrm{np})=\operatorname{zvecs}(\mathrm{nx}, \mathrm{ny}, \mathrm{nz}, \mathrm{nt}, \mathrm{ns}, \mathrm{ni}, \mathrm{np}) \&$

$+\mathrm{w} 3 \mathrm{~N} * \mathrm{~h} * \mathrm{zvecs}(\mathrm{MOD}(\mathrm{nx}+3, \mathrm{~L}), \mathrm{ny}, \mathrm{nz}, \mathrm{nt}-1, \mathrm{~ns}, \mathrm{ni}, \mathrm{np}) \&$

+ w3_N*h*zvecs (MOD(nx-3+L, L) ,ny, nz, nt-1,ns, ni,np) \&

+ w3_N*h*zvecs ( nx ,MOD(ny+3,L), nz, nt-1,ns, ni, np) \&

+ w3_N*h*zvecs (nx,MOD(ny-3+L, L), nz, nt-1,ns, ni,np) \&

+ w3_N*h*zvecs ( nx, ny, MOD (nz+3,L), nt-1,ns, ni, np) \&

END IF

+ w3_N*h*zvecs (nx, ny,MOD(nz-3+L,L), nt-1,ns, ni ,np)

\section{END DO; END DO; END DO; END DO; END DO \\ END DO \\ END DO}

Similarly, we now show sample code 6.2 which calculates the auxiliary-field transfer matrix multiplications on the right starting from the single-nucleon final states. Again we present only the terms arising from the free-nucleon transfer matrix and the auxiliary field $s$.

Listing 6.2 Sample code calculating the auxiliary-field transfer matrix multiplications on the right starting from the single-nucleon final states

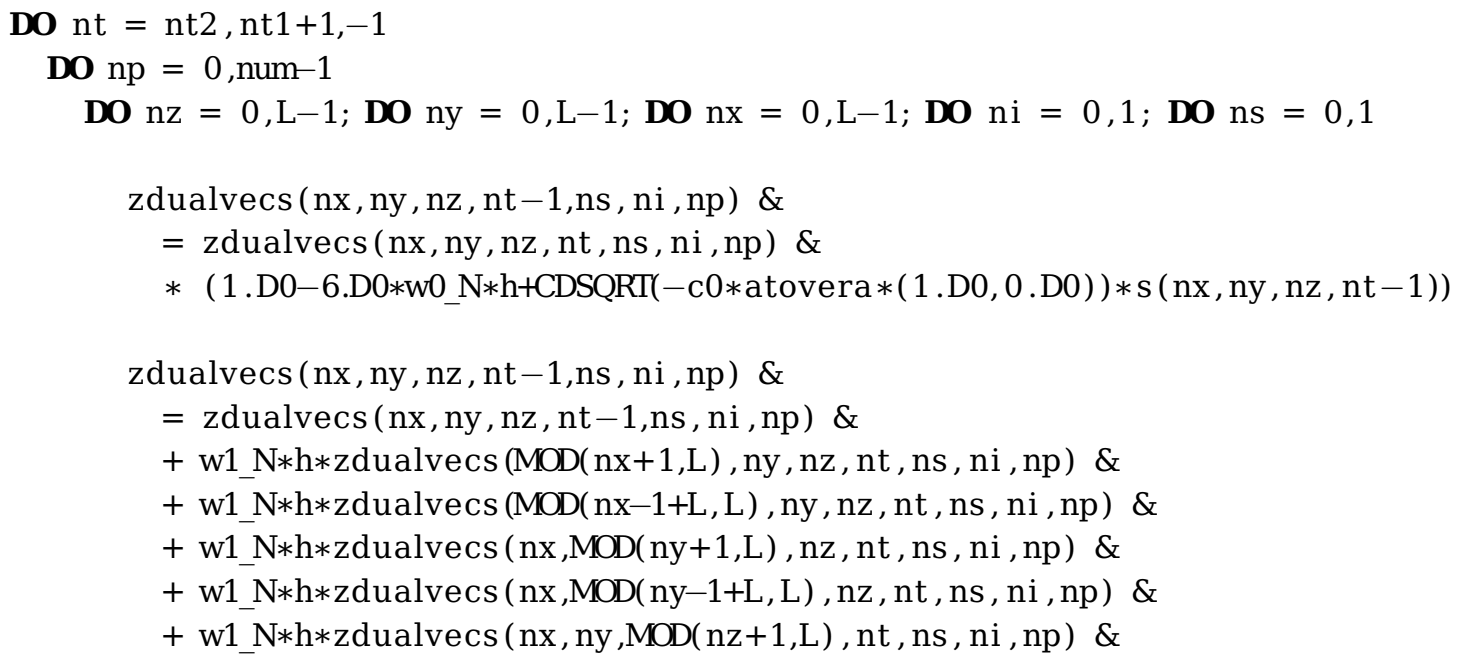




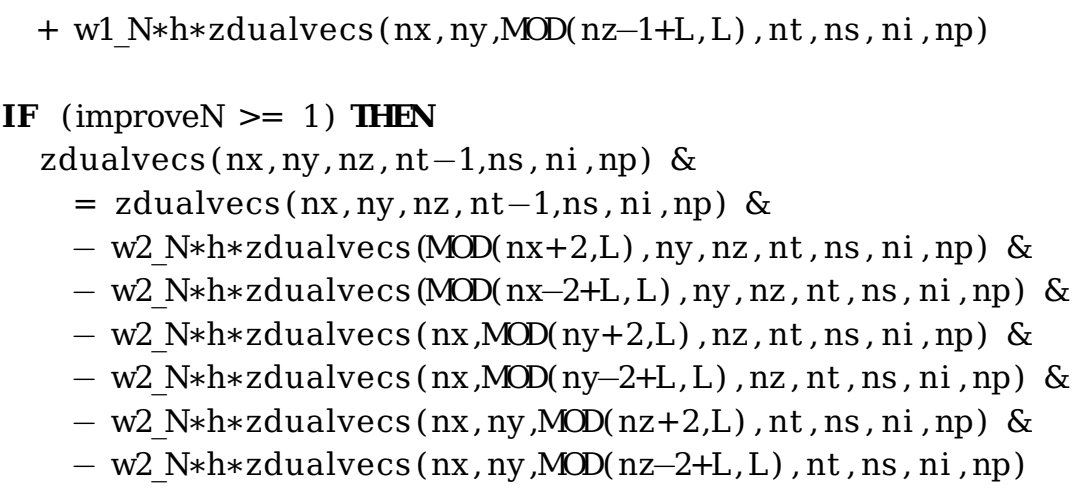

\section{END IF}

IF (improveN $==2$ ) THEN

zdualvecs (nx, ny, nz, nt-1,ns, ni ,np) \&

$=$ zdualvecs $(\mathrm{nx}, \mathrm{ny}, \mathrm{nz}, \mathrm{nt}-1, \mathrm{~ns}, \mathrm{ni}, \mathrm{np}) \&$

+ w3_N*h*zdualvecs (MOD(nx+3,L), ny, nz, nt, ns, ni, np) \&

+ w3_N*h*zdualvecs (MOD(nx-3+L, L) , ny, nz, nt, ns, ni, np) \&

+ w3_N*h*zdualvecs ( $\mathrm{nx}, \mathrm{MOD}(\mathrm{ny}+3, \mathrm{~L}), \mathrm{nz}, \mathrm{nt}, \mathrm{ns}, \mathrm{ni}, \mathrm{np}) \&$

+ w3_N*h*zdualvecs ( $\mathrm{nx}, \mathrm{MOD}(\mathrm{ny}-3+\mathrm{L}, \mathrm{L}), \mathrm{nz}, \mathrm{nt}, \mathrm{ns}, \mathrm{ni}, \mathrm{np}) \&$

+ w3_N*h*zdualvecs ( $n x, n y, M O D(n z+3, L), n t, n s, n i, n p) \&$

+ w3_N*h*zdualvecs (nx, ny, MOD $(n z-3+L, L), n t, n s, n i, n p)$

\section{END IF}

\section{END DO; END DO \\ END DO; END DO; END DO END DO}

In the following we show sample code 6.3 where these transfer matrix product multiplications are called as subroutines and used to compute the determinant and inverse of the matrix of single-nucleon amplitudes $\mathbf{M}$.

Listing 6.3 Sample code where transfer matrix product multiplications are called and used to compute the determinant and inverse of the matrix of single-nucleon amplitudes.

CALL getzvecs ( $\mathrm{s}, \mathrm{sI}$, zvecs , zwave, Lt , 0 , pion , ztau2x2, n_f )

CALL getzdualvecs ( $\mathrm{s}, \mathrm{sI}, \mathrm{zdualvecs}, \mathrm{zdualwave}, \mathrm{Lt}, 0$, pion, ztau2 $\mathrm{x} 2, \mathrm{n} \mathrm{f}$ )

CALL getinvcorr (zvecs, zdualvecs, zldeter , zcorrmatrix , zcorrinv , Lt)

aldeterabs $=$ DBLE $($ zldeter $)$

zdeterphase $=\operatorname{CDEXP}((0 . \mathrm{D} 0,1 . \mathrm{D} 0) * \operatorname{DIMAG}($ zldeter $))$

act $=$ bose - aldeterabs

\subsection{Importance sampling}

We do importance sampling according to the positive measure

$$
\left|Z\left(L_{t}\right)\right| \exp \left[-S_{s S}(s)-S_{S_{I} S_{I}}\left(s_{I}\right)-S_{\pi_{I} \pi_{I}}\left(\pi_{I}\right)\right],
$$

and use hybrid Monte Carlo to do global updates of the auxiliary and pion fields. The hybrid Monte Carlo (HMC) algorithm [15 17] is efficient in quickly generating decorrelated configurations for each auxiliary and pion field. Here we describe the updating algorithm for the $s$ 
field. The updating of the $s_{I}$ and $\pi_{I}$ fields proceed in a very similar fashion. In general terms, the HMC algorithm can be described by means of a probability weight $P(s)$

$$
P(s) \propto \exp [-V(s)]
$$

where $V(s)$ is in general a non-local function of the field $s\left(\mathbf{n}, n_{t}\right)$, and a molecular dynamics (MD) Hamiltonian,

$$
H(s, p) \equiv \frac{1}{2} \sum_{\mathbf{n}, n_{t}}\left[p_{s}\left(\mathbf{n}, n_{t}\right)\right]^{2}+V(s) .
$$

Classical Hamiltonian dynamics is introduced by defining the momentum $p_{s}\left(\mathbf{n}, n_{t}\right)$ conjugate to $s\left(\mathbf{n}, n_{t}\right)$.

Given an arbitrary initial configuration $s^{0}\left(\mathbf{n}, n_{t}\right)$, the conjugate momentum is chosen from a random Gaussian distribution according to

$$
P\left[p_{s}^{0}\left(\mathbf{n}, n_{t}\right)\right] \propto \exp \left\{-\frac{1}{2}\left[p_{s}^{0}\left(\mathbf{n}, n_{t}\right)\right]^{2}\right\},
$$

after which the Hamiltonian equations of motion are integrated numerically with a small but nonzero step size $\varepsilon_{\text {step }}$. This method begins with a "half-step" forward in the conjugate momentum,

$$
\tilde{p}_{s}^{0}\left(\mathbf{n}, n_{t}\right)=p_{s}^{0}\left(\mathbf{n}, n_{t}\right)-\frac{\varepsilon_{\text {step }}}{2}\left[\frac{\partial V(s)}{\partial s\left(\mathbf{n}, n_{t}\right)}\right]_{s=s^{0}},
$$

followed by repeated updates of $s$ and $\tilde{p}_{s}$ according to

$$
s^{i+1}\left(\mathbf{n}, n_{t}\right)=s^{i}\left(\mathbf{n}, n_{t}\right)+\varepsilon_{\text {step }} \tilde{p}_{s}^{i}\left(\mathbf{n}, n_{t}\right), \quad \tilde{p}_{s}^{i+1}\left(\mathbf{n}, n_{t}\right)=\tilde{p}_{s}^{i}\left(\mathbf{n}, n_{t}\right)-\varepsilon_{\text {step }}\left[\frac{\partial V(s)}{\partial s\left(\mathbf{n}, n_{t}\right)}\right]_{s=s^{i+1}},
$$

for a specified number of steps $N_{\text {step }}$. This is followed by an additional half-step backward in $\tilde{p}_{s}$ given by

$$
p_{s}^{N_{\text {step }}}\left(\mathbf{n}, n_{t}\right)=\tilde{p}_{s}^{N_{\text {step }}}\left(\mathbf{n}, n_{t}\right)+\frac{\varepsilon_{\text {step }}}{2}\left[\frac{\partial V(s)}{\partial s\left(\mathbf{n}, n_{t}\right)}\right]_{s=s^{0}} .
$$

For algorithmic efficiency the length of such an MD "trajectory" should be taken large enough to ensure decorrelation between successive configurations of the auxiliary field. The evolved configuration is then subjected to a "Metropolis test" against a random number $r \in$ $[0,1)$. The new configuration is accepted if

$$
r<\exp \left[-H\left(s^{N_{\text {step }}}, p_{s}^{N_{\text {step }}}\right)+H\left(s^{0}, p_{s}^{0}\right)\right] .
$$

It should be noted that although $H$ is in principle conserved in the MD evolution, the truncation error of the leapfrog method introduces a systematic error. The Metropolis test eliminates the need for extrapolation in $\varepsilon_{\text {step }}$.

In our case $\exp [-V(s)]$ has the form

$$
\left|Z\left(L_{t}\right)\right| \exp \left[-S_{s s}(s)-S_{S_{I} S_{I}}\left(s_{I}\right)-S_{\pi_{I} \pi_{I}}\left(\pi_{I}\right)\right],
$$

where $Z\left(L_{t}\right)$ is the determinant of an $A \times A$ matrix of single-nucleon amplitudes $\mathbf{M}$. The derivative of $V$ is then computed using 


$$
\begin{aligned}
\frac{\partial V(s)}{\partial s\left(\mathbf{n}, n_{t}\right)} & =\frac{\partial S_{s s}(s)}{\partial s\left(\mathbf{n}, n_{t}\right)}-\frac{\partial \operatorname{Re}[\ln (\operatorname{det} \mathbf{M})]}{\partial s\left(\mathbf{n}, n_{t}\right)} \\
& =\frac{\partial S_{s s}(s)}{\partial s\left(\mathbf{n}, n_{t}\right)}-\operatorname{Re}\left[\frac{1}{\operatorname{det} \mathbf{M}} \sum_{k, l} \frac{\partial \operatorname{det} \mathbf{M}}{\partial \mathbf{M}_{k l}} \frac{\partial \mathbf{M}_{k l}}{\partial s\left(\mathbf{n}, n_{t}\right)}\right] \\
& =\frac{\partial S_{s s}(s)}{\partial s\left(\mathbf{n}, n_{t}\right)}-\operatorname{Re}\left[\sum_{k, l} \mathbf{M}_{l k}^{-1} \frac{\partial \mathbf{M}_{k l}}{\partial s\left(\mathbf{n}, n_{t}\right)}\right] .
\end{aligned}
$$

In the following we show sample code 6.4 calculating the quadratic part of the action due to the auxiliary fields and pion fields,

$$
\frac{1}{2} \sum_{\mathbf{n}, n_{t}}\left[p_{s}\left(\mathbf{n}, n_{t}\right)\right]^{2}+\frac{1}{2} \sum_{\mathbf{n}, n_{t}, I}\left[p_{s_{I}}\left(\mathbf{n}, n_{t}\right)\right]^{2}+\frac{1}{2} \sum_{\mathbf{n}, n_{t}, I}\left[p_{\pi_{I}}\left(\mathbf{n}, n_{t}\right)\right]^{2}+S_{s s}(s)+S_{S_{I} S_{I}}\left(s_{I}\right)+S_{\pi_{I} \pi_{I}}\left(\pi_{I}\right) .
$$

In the code we have found it convenient to rescale the pion field by a factor of $\sqrt{q_{\pi}}$ where

$$
q_{\pi}=\alpha_{t}\left(m_{\pi}^{2}+6 w_{0}\right) .
$$

Listing 6.4 Sample code calculating the quadratic part of the action due to the auxiliary fields and pion fields.

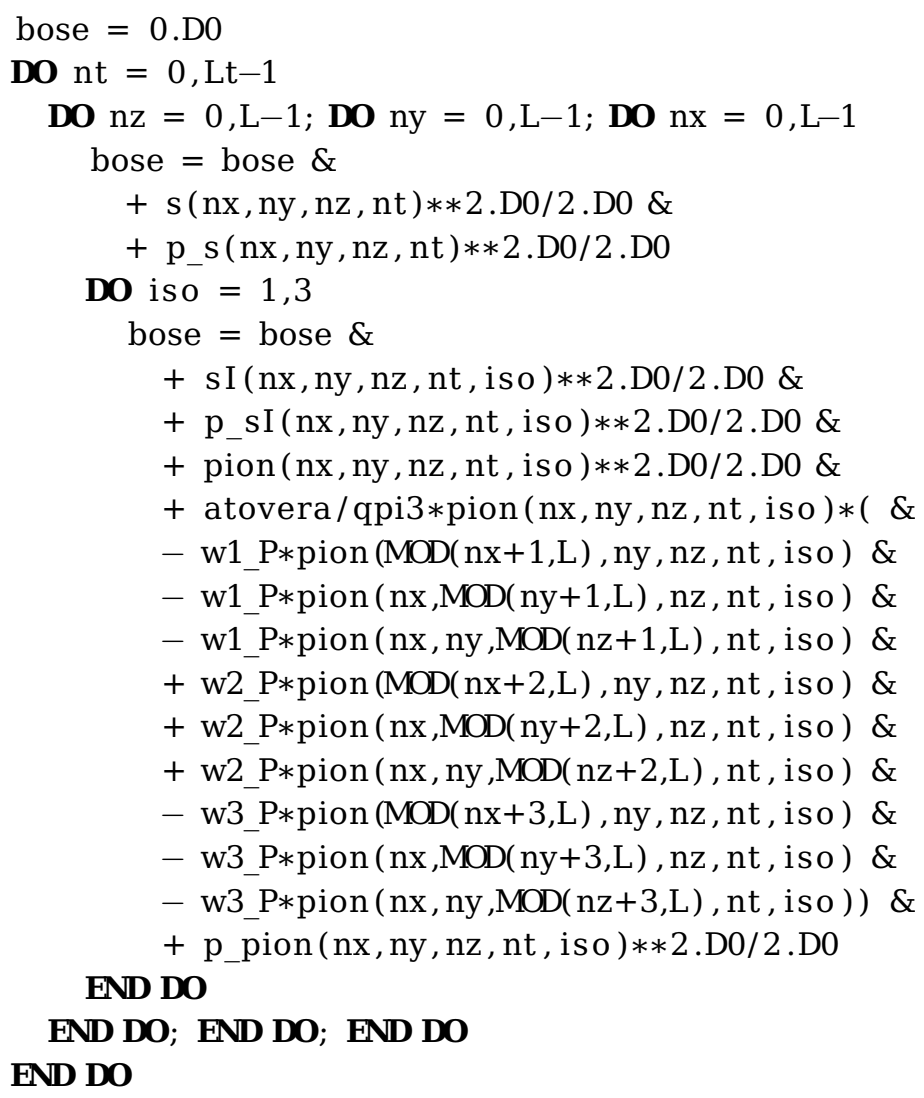

In following we show sample code 6.5 which calculates

$$
\left[\frac{\partial V(s)}{\partial s\left(\mathbf{n}, n_{t}\right)}\right]_{s=s^{0}}
$$

and uses it to compute the half-step forward in the conjugate momentum, 


$$
\tilde{p}_{s}^{0}\left(\mathbf{n}, n_{t}\right)=p_{s}^{0}\left(\mathbf{n}, n_{t}\right)-\frac{\varepsilon_{\text {step }}}{2}\left[\frac{\partial V(s)}{\partial s\left(\mathbf{n}, n_{t}\right)}\right]_{s=s^{0}} .
$$

Listing 6.5 Sample code computing derivative with respect to the auxiliary field and half-step forward in the conjugate momentum.

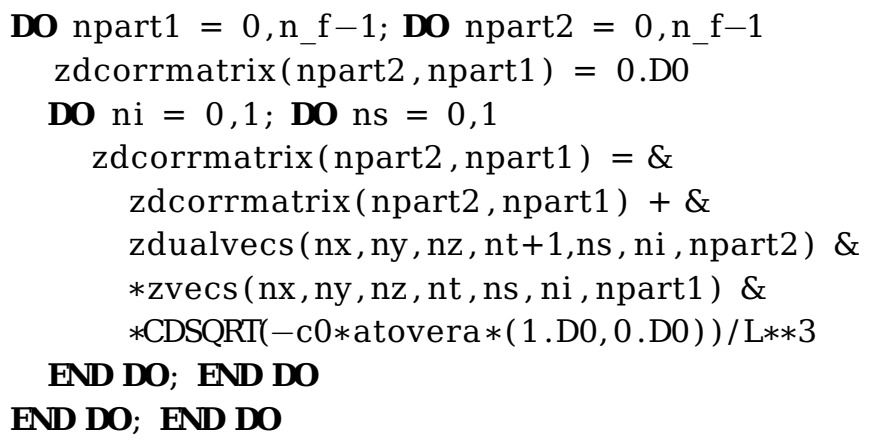

\section{END DO; END DO}

$\mathrm{p} \_\operatorname{sHMC}(\mathrm{nx}, \mathrm{ny}, \mathrm{nz}, \mathrm{nt}, 0)=\&$

p_s $(\mathrm{nx}, \mathrm{ny}, \mathrm{nz}, \mathrm{nt})-0.5 \mathrm{D} 0 * \mathrm{eHMC} * \mathrm{dVds}(\mathrm{nx}, \mathrm{ny}, \mathrm{nz}, \mathrm{nt})$

In following code 6.6 we show an example code which performs the Metropolis test against a random number $r \in[0,1)$, with the new configuration being accepted if

$$
r<\exp \left[-H\left(s^{N_{\text {step }}}, p_{s}^{N_{\text {step }}}\right)+H\left(s^{0}, p_{s}^{0}\right)\right] .
$$

Listing 6.6 Sample code which performs the Metropolis acceptance test

IF (ntrial .eq. 1 .or. grnd() . lt. DEXP(-actnew+act)) THEN

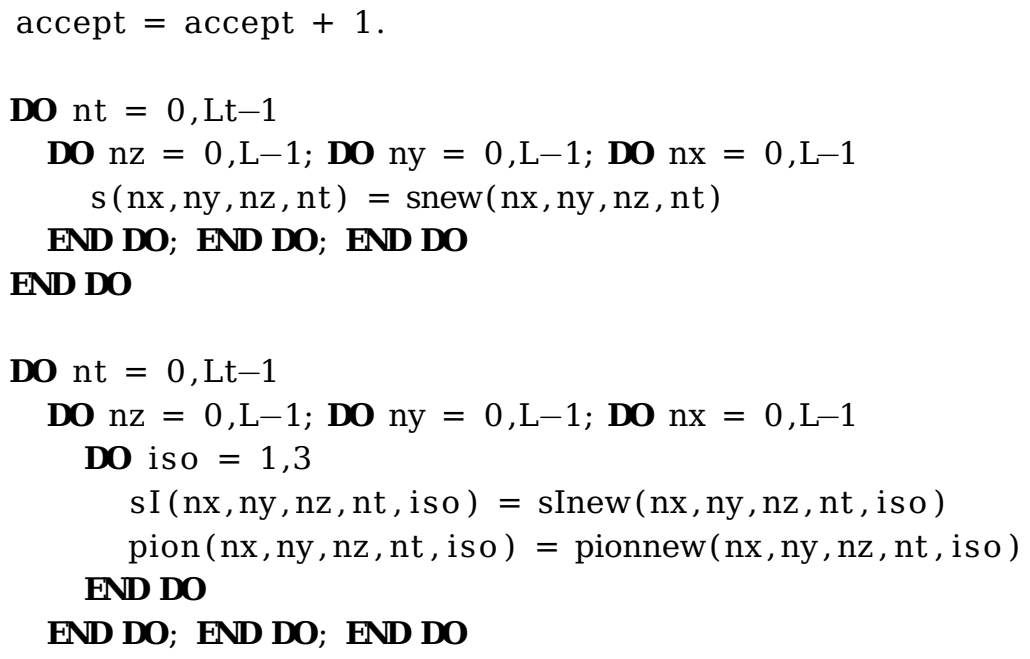


END DO

aldeterabs $=$ aldeternewabs

zdeterphase $=$ zdeternewphase

END IF

Although the Monte Carlo importance sampling uses only the absolute value of the amplitude, the complex phase of the amplitude is treated as an observable and is collected with each configuration of the auxiliary and pion fields.

\subsection{Exercises}

6.1. Write a lattice hybrid Monte Carlo code which performs updates of the lattice action according to only the quadratic part of the action due to the auxiliary fields and pions,

$$
\frac{1}{2} \sum_{\mathbf{n}, n_{t}}\left[p_{s}\left(\mathbf{n}, n_{t}\right)\right]^{2}+\frac{1}{2} \sum_{\mathbf{n}, n_{t}, I}\left[p_{S_{I}}\left(\mathbf{n}, n_{t}\right)\right]^{2}+\frac{1}{2} \sum_{\mathbf{n}, n_{t}, I}\left[p_{\pi_{I}}\left(\mathbf{n}, n_{t}\right)\right]^{2}+S_{s s}(s)+S_{S_{I} S_{I}}\left(s_{I}\right)+S_{\pi_{I} \pi_{I}}\left(\pi_{I}\right) .
$$

Verify that the change in the action produced by the hybrid Monte Carlo update is scaling quadratically in the step size, $\varepsilon_{\text {step }}$, in the limit $\varepsilon_{\text {step }} \rightarrow 0$ with $N_{\text {step }} \varepsilon_{\text {step }}$ held fixed.

6.2. Write a function or subroutine that generates initial/final single-nucleon states on the lattice corresponding to a Slater-determinant state with one neutron spin-up and one neutron spin-down, both with zero momentum.

6.3. Write a function or subroutine that generates initial/final single-nucleon states on the lattice corresponding to a Slater-determinant state with one proton spin-up and one neutron spin-up, both with zero momentum.

6.4. Write a function or subroutine that generates initial/final single-nucleon states on the lattice corresponding to a Slater-determinant state of four nucleons - proton spin-up, proton spin-down, neutron spin-up, and neutron spin-down - each with zero momentum.

6.5. Write a function or subroutine that extends the sample code 6.1 to repeatedly multiply the auxiliary-field transfer matrix on the left starting from the initial single-nucleon wave functions. Include the contributions from the auxiliary fields $s$ and $s_{I}$ as well as the pion field $\pi_{I}$.

6.6. Write a function or subroutine that extends the sample code 6.2 to repeatedly multiply the auxiliary-field transfer matrix on the right starting from the final single-nucleon wave functions. Include the contributions from the auxiliary fields $s$ and $s_{I}$ as well as the pion field $\pi_{I}$.

6.7. Use the Slater-determinant states constructed in Probs. 6.2, 6.3, 6.4 as initial and final states. In each case apply the functions or subroutines written in Prob. 6.5 and Prob. 6.6 with all coupling constants set to zero. Verify that in each case the initial/final state is the ground state of the non-interacting system with energy equal to zero.

6.8. Use the Slater-determinant states constructed in Probs. 6.2, 6.3, 6.4 as initial and final states. Using the functions or subroutines written in Prob. 6.5 and Prob. 6.6, extend the sample code 6.5 to compute the derivatives of $V(s)$ with respect to $s\left(\mathbf{n}, n_{t}\right), s_{I}\left(\mathbf{n}, n_{t}\right)$, and $\pi_{I}\left(\mathbf{n}, n_{t}\right)$. 
6.9. Take the code you have written for Prob. 6.8 and complete the remaining steps needed to do hybrid Monte Carlo updates for $s, s_{I}$, and $\pi_{I}$. Verify that the change in the action produced by the hybrid Monte Carlo update is scaling quadratically in $\varepsilon_{\text {step }}$ in the limit $\varepsilon_{\text {step }} \rightarrow 0$ with $N_{\text {step }} \varepsilon_{\text {step }}$ held fixed.

6.10. Take the code you have written for Prob. 6.9 and complete the remaining steps needed to calculate the energy of the ground state by computing the ratio of the amplitudes $Z\left(L_{t}\right) / Z\left(L_{t}-1\right)$.

\subsection{Codes and Benchmarks}

Complete verisons of the codes discussed in this chapter and developed in the exercises can be found online via this link. In order to run the codes, one must first copy the corresponding initial/final wavefunctions (waveinit_1S0.f90, waveinit_3S1.f90, or waveinit_He4.f90) into the file waveinit.f90 used by the main program nuclei.f90. The number of nucleons is controlled by the parameter $\mathrm{n}_{-} \mathrm{f}$ in input.f90 and must correspond to the number of nucleons in waveinit.f90.

As an example we show the beginning of the input file input.f90 for a two nucleon state with spatial lattice spacing $a=1 /(100 \mathrm{MeV})$, temporal lattice spacing $a_{t}=1 /(150 \mathrm{MeV})$, box size $L=4 a$, and Euclidean time extent $L_{t}=6 a_{t}$. We use an $O\left(a^{4}\right)$-improved lattice action for the nucleon hopping coefficients, $O\left(a^{0}\right)$-improved lattice action for the pion hopping coefficients, and $O\left(a^{0}\right)$-improved lattice action for the pion-nucleon coupling. The coefficient of the ${ }^{1} S_{0}$ contact interaction is tuned to the physical ${ }^{1} S_{0} n-p$ scattering length and is $-5.615 \times 10^{-5} \mathrm{MeV}^{-2}$. The coefficient of the ${ }^{3} S_{1}$ contact interaction is tuned to the deuteron binding energy at infinite volume and is $-6.543 \times 10^{-5} \mathrm{MeV}^{-2}$.

Listing 6.7 Parameter declarations at the beginning of the file input.f90.

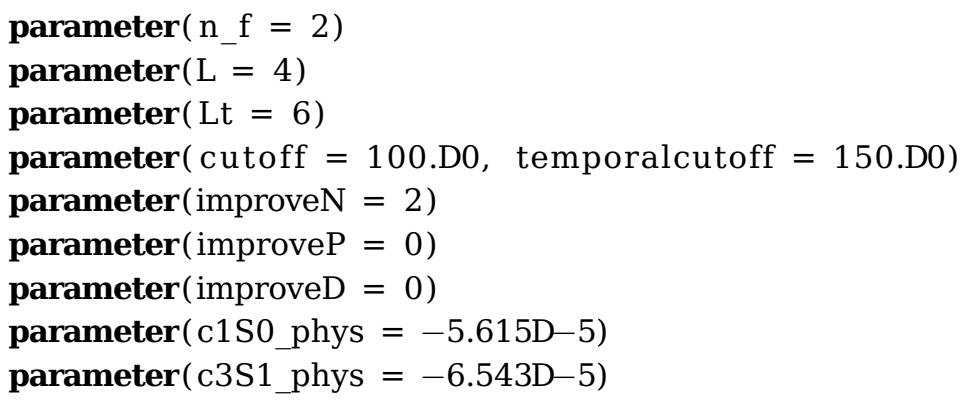

Using these values for the parameters of the lattice action, we now present some benchmark values which can be used to test the nuclear lattice simulations in the two-nucleon system. The values presented in these benchmarks are computed using exact calculations of the two-nucleon transfer matrix. They provide a useful independent check that there are no errors in the Monte Carlo simulations. In Table 6.4 we show the energies for the ${ }^{1} S_{0}$ spin combination of two nucleons. The initial state is one neutron spin-up and one neutron spin-down, both at zero momentum, for $L=4 a$ and various values of $L_{t}$. The energies are extracted by computing the ratio of amplitudes $Z\left(L_{t}\right) / Z\left(L_{t}-1\right)$ and setting equal to $\exp \left(-E \alpha_{t}\right)$.

We show the energies for the ${ }^{3} S_{1}$ spin combination of two nucleons in Table 6.5. The initial state is one proton spin-up and one neutron spin-up, both at zero momentum, for $L=4 a$ and various values of $L_{t}$. The energies are extracted by computing the ratio of amplitudes $Z\left(L_{t}\right) / Z\left(L_{t}-1\right)$ and setting equal to $\exp \left(-E \alpha_{t}\right)$. 
Table 6.4 Benchmark energies for the ${ }^{1} S_{0}$ spin combination of two nucleons. The initial state is one neutron spin-up and one neutron spin-down, both at zero momentum, for $L=4 a$ and various values of $L_{t}$.

\begin{tabular}{ll}
\hline$L_{t}$ & energy $(\mathrm{MeV})$ \\
\hline 2 & -1.0915 \\
4 & -1.3987 \\
6 & -1.6209 \\
8 & -1.7929 \\
10 & -1.9296 \\
12 & -2.0398 \\
14 & -2.1291 \\
16 & -2.2018 \\
18 & -2.2610 \\
20 & -2.3094 \\
\hline
\end{tabular}

Table 6.5 Benchmark energies for the ${ }^{3} S_{1}$ spin combination of two nucleons. The initial state is one proton spin-up and one neutron spin-up, both at zero momentum, for $L=4 a$ and various values of $L_{t}$.

\begin{tabular}{ll}
\hline$L_{t}$ & energy $(\mathrm{MeV})$ \\
\hline 2 & -1.4446 \\
4 & -2.0400 \\
6 & -2.4774 \\
8 & -2.8331 \\
10 & -3.1341 \\
12 & -3.3925 \\
14 & -3.6151 \\
16 & -3.8069 \\
18 & -3.9718 \\
20 & -4.1132 \\
\hline
\end{tabular}

Acknowledgements The author is grateful for discussions with Amy Nicholson and Morten Hjorth-Jensen. $\mathrm{He}$ is also greatly indebted to his collaborators Jose Alarcón, Dechuan Du, Serdar Elhatisari, Evgeny Epelbaum, Nico Klein, Hermann Krebs, Timo Lähde, Ning Li, Bing-nan Lu, Thomas Luu, Ulf-G. Meißner, Alexander Rokash, and Gautam Rupak. Partial financial support provided by the U.S. Department of Energy (DE-FG0203ER41260). Computational resources were provided by the Jülich Supercomputing Centre.

\section{References}

1. S. Weinberg, Phys. Lett. B251, 288 (1990)

2. S. Weinberg, Nucl. Phys. B363, 3 (1991)

3. C. Ordonez, U. van Kolck, Phys. Lett. B291, 459 (1992)

4. C. Ordonez, L. Ray, U. van Kolck, Phys. Rev. Lett. 72, 1982 (1994)

5. U. van Kolck, Phys. Rev. C49, 2932 (1994)

6. E. Epelbaum, W. Glöckle, U.G. Meißner, Phys. Lett. B439, 1 (1998)

7. E. Epelbaum, W. Glöckle, U.G. Meißner, Nucl. Phys. A637, 107 (1998)

8. P.F. Bedaque, U. van Kolck, Ann. Rev. Nucl. Part. Sci. 52, 339 (2002)

9. E. Epelbaum, H.W. Hammer, U.G. Meißner, Rev. Mod. Phys. 81, 1773 (2009)

10. D. Lee, Prog. Part. Nucl. Phys. 63, 117 (2009)

11. J.E. Drut, A.N. Nicholson, J. Phys. G: Nucl. Part. Phys. 40(4), 043101 (2013). DOI 10.1088/0954-3899/40/ $4 / 043101$

12. J. Hubbard, Phys. Rev. Lett. 3, 77 (1959)

13. R.L. Stratonovich, Soviet Phys. Doklady 2, 416 (1958)

14. D.H. Weingarten, D.N. Petcher, Phys. Lett. B99, 333 (1981) 
15. R.T. Scalettar, D.J. Scalapino, R.L. Sugar, Phys. Rev. B34, 7911 (1986)

16. S. Gottlieb, W. Liu, D. Toussaint, R.L. Renken, R.L. Sugar, Phys. Rev. D35, 2531 (1987)

17. S. Duane, A.D. Kennedy, B.J. Pendleton, D. Roweth, Phys. Lett. B195, 216 (1987)

18. H.M. Müller, S.E. Koonin, R. Seki, U. van Kolck, Phys. Rev. C61, 044320 (2000)

19. D. Lee, B. Borasoy, T. Schäfer, Phys. Rev. C70, 014007 (2004)

20. D. Lee, T. Schäfer, Phys. Rev. C72, 024006 (2005)

21. D. Lee, T. Schäfer, Phys. Rev. C73, 015201 (2006)

22. D. Lee, T. Schäfer, Phys. Rev. C73, 015202 (2006)

23. B. Borasoy, H. Krebs, D. Lee, U.G. Meißner, Nucl. Phys. A768, 179 (2006)

24. B. Borasoy, E. Epelbaum, H. Krebs, D. Lee, U.G. Meißner, Eur. Phys. J. A31, 105 (2007)

25. B. Borasoy, E. Epelbaum, H. Krebs, D. Lee, U.G. Meißner, Eur. Phys. J. A35, 343 (2008)

26. B. Borasoy, E. Epelbaum, H. Krebs, D. Lee, U.G. Meißner, Eur. Phys. J. A35, 357 (2008)

27. G. Wlazłowski, J.W. Holt, S. Moroz, A. Bulgac, K.J. Roche, Phys. Rev. Lett. 113(18), 182503 (2014). DOI 10.1103/PhysRevLett.113.182503

28. E. Epelbaum, H. Krebs, D. Lee, U.G. Meißner, Eur. Phys. J. A41, 125 (2009)

29. E. Epelbaum, H. Krebs, D. Lee, U.G. Meißner, Phys. Rev. Lett. 104, 142501 (2010)

30. E. Epelbaum, H. Krebs, D. Lee, U.G. Meißner, Phys. Rev. Lett. 106, 192501 (2011). DOI 10.1103/ PhysRevLett.106.192501

31. E. Epelbaum, H. Krebs, T. Lähde, D. Lee, U.G. Meißner, Phys. Rev. Lett. 109, 252501 (2012). DOI 10.1103/PhysRevLett.109.252501

32. E. Epelbaum, H. Krebs, T.A. Lähde, D. Lee, U.G. Meißner, Phys. Rev. Lett. 110, 112502 (2013). DOI 10.1103/PhysRevLett.110.112502

33. T.A. Lähde, E. Epelbaum, H. Krebs, D. Lee, U.G. Meißner, G. Rupak, Phys. Lett. B732, 110 (2014). DOI 10.1016/j.physletb.2014.03.023

34. E. Epelbaum, H. Krebs, T.A. Lähde, D. Lee, U.G. Meißner, G. Rupak, Phys. Rev. Lett. 112(10), 102501 (2014). DOI 10.1103/PhysRevLett.112.102501

35. S. Elhatisari, et al., (2016)

36. G. Rupak, D. Lee, Phys. Rev. Lett. 111(3), 032502 (2013). DOI 10.1103/PhysRevLett.111.032502

37. G. Rupak, P. Ravi, Phys. Lett. B741, 301 (2014). DOI 10.1016/j.physletb.2014.12.055

38. S. Elhatisari, D. Lee, G. Rupak, E. Epelbaum, H. Krebs, T.A. Lähde, T. Luu, U.G. Meißner, Nature 528, 111 (2015). DOI 10.1038/nature16067

39. A. Ekström, G.R. Jansen, K.A. Wendt, G. Hagen, T. Papenbrock, B.D. Carlsson, C. Forssen, M. HjorthJensen, P. Navratil, W. Nazarewicz, Phys. Rev. C91(5), 051301 (2015). DOI 10.1103/PhysRevC.91.051301

40. G. Hagen, et al., Nature Phys. (2015). DOI 10.1038/nphys3529

41. M. Lüscher, Commun. Math. Phys. 104, 177 (1986)

42. M. Lüscher, Commun. Math. Phys. 105, 153 (1986)

43. M. Lüscher, Nucl. Phys. B354, 531 (1991)

44. B. Borasoy, E. Epelbaum, H. Krebs, D. Lee, U.G. Meißner, Eur. Phys. J. A34, 185 (2007)

45. J. Carlson, V. Pandharipande, R. Wiringa, Nucl. Phys. A 424(1), 47 (1984). DOI http://dx. doi.org/10.1016/0375-9474(84)90127-1. URL http://www. sciencedirect.com/science/article/pii/ 0375947484901271

46. B.N. Lu, T.A. Lähde, D. Lee, U.G. Meißner, (2015)

47. M. Pine, D. Lee, G. Rupak, Eur. Phys. J. A49, 151 (2013). DOI 10.1140/epja/i2013-13151-3

48. S. Elhatisari, D. Lee, Phys. Rev. C90(6), 064001 (2014). DOI 10.1103/PhysRevC.90.064001

49. A. Rokash, M. Pine, S. Elhatisari, D. Lee, E. Epelbaum, et al., (2015)

50. M. Creutz, Phys. Rev. D38, 1228 (1988)

51. M. Creutz, Found. Phys. 30, 487 (2000)

52. J. Carlson, S. Gandolfi, F. Pederiva, S.C. Pieper, R. Schiavilla, K.E. Schmidt, R.B. Wiringa, Rev. Mod. Phys. 87, 1067 (2015). DOI 10.1103/RevModPhys.87.1067 